\title{
The modified Medical Research Council dyspnoea scale is a good indicator of health-related quality of life in patients with chronic obstructive pulmonary disease
}

\author{
Kun-Yen $\underline{H s u}^{1,3}$, MD, Jr-Rung $\underline{L i n}^{1,2}$, PhD, Ming-Shian Lin ${ }^{3}, M D$, Wei Chen ${ }^{3}$, MD, Yi-Jen Chen ${ }^{3}$, MD,
} Yuan-Horng $\underline{\mathrm{Yan}}^{4}$, MD

INTRODUCTION Health-related quality of life ( $\mathrm{HRQOL}$ ) is an important patient-centred outcome in chronic obstructive pulmonary disease (COPD). The aim of the current study is to compare the discriminative capacity of the modified Medical Research Council (mMRC) dyspnoea scale and the Global Initiative for Chronic Obstructive Lung Disease (GOLD) spirometric classification of COPD on HRQOL, as well as determine other factors that are simple and determinative of HRQOL.

METHODS In this cross-sectional observational study, a total of 328 patients with COPD were enrolled from the pulmonology outpatient clinic. HRQoL was measured using the St George's Respiratory Questionnaire (SGRQ) and the World Health Organization Quality of Life-BREF (WHOQOL-BREF). HRQoL scores were compared between the four GOLD stages and the five grades of the mMRC scale. Significant differences were determined using analysis of variance with Scheffe post-hoc test. Multiple linear regression was applied to explore the major determinants of HRQOL and exclude confounding factors.

RESULTS Significant differences were found in many more domains of the two questionnaires between mMRC grades than between GOLD stages. In the multiple linear regression model, the mMRC scale was the only factor that remained determinative of all the domains of SGRQ and WHOQOL-BREF. Patients with chronic productive cough, sleep disorders and frequent exacerbations had poorer HRQoL, as reflected by higher scores in SGRQ or lower Scores in WHOQOL-BREF.

CONCLUSION The mMRC dyspnoea scale is a concise and practical tool to assess the HRQL of patients with COPD in daily clinical practice.

Keywords: chronic obstructive pulmonary disease, Global Initiative for Chronic Obstructive Lung Disease, health-related quality of life, Medical Research Council dyspnoea scale, spirometry

\section{INTRODUCTION}

Chronic obstructive pulmonary disease (COPD), a chronic respiratory disease with high prevalence worldwide, is becoming a leading cause of mortality and morbidity. ${ }^{(1)}$ Healthrelated quality of life (HRQoL) is a patient-reported outcome that is receiving increasing recognition regarding its use in patients with COPD. ${ }^{(2,3)}$ HRQoL offers an integral measurement of overall health status and the impact of disease on patients. In COPD clinical trials, HRQoL is frequently included as an endpoint to evaluate the effects of pharmacological and non-pharmacological treatments. ${ }^{(4-7)}$ In addition, HRQoL also predicts mortality and hospitalisation in patients with COPD. ${ }^{(2,8,9)}$ HRQoL is generally measured using validated questionnaires, either disease-specific or generic, and a comprehensive evaluation of HRQoL should enclose both disease-specific and generic instruments. ${ }^{(10)}$ However, since the application of HRQoL questionnaires in daily clinical practice is often restricted due to time constraints, a simple surrogate marker that is discriminative of HRQoL would be helpful.
A spirometric classification of COPD (Stages 1 to 4), based on forced expiratory volume in 1 second $\left(\mathrm{FEV}_{1}\right)$ percentage predicted, had been advocated by the Global Initiative for Chronic Obstructive Lung Disease (GOLD). However, the cutoff points recommended are used for the purpose of simplicity and have not been clinically validated. ${ }^{(1)}$ Furthermore, FEV $_{1}$ is often poorly correlated with patient-centred outcomes such as HRQoL. ${ }^{(11-13)}$

The modified Medical Research Council (mMRC) scale is a five-level rating scale based on the patient's perception of dyspnoea in daily activities. ${ }^{(14)}$ For patients with COPD, it is a simple and valid tool to assess disability, ${ }^{(15)}$ and has been reported to be more relevant to patients' health and psychological status than $\mathrm{FEV}_{1}{ }^{\left({ }^{(16)}\right.}$ To the best of our knowledge, since the GOLD spirometric classification was published, no study has reported direct comparisons between these two indices to categorise patients with COPD with regard to HRQoL.

In this cross-sectional observational study, we compared the discriminative capacity of the GOLD spirometric classification

\footnotetext{
${ }^{1}$ Graduate Institute of Clinical Medical Sciences, College of Medicine, ${ }^{2}$ Clinical Informatics and Medical Statistics Research Center, Chang Gung University, Tao-Yuan, Taiwan, ${ }^{3}$ Division of Pulmonary and Critical Care Medicine, Department of Internal Medicine, ${ }^{4}$ Department of Medical Research, Chia-Yi Christian Hospital, Chia-Yi, Taiwan

Correspondence: Dr Jr-Rung Lin, Assistant Professor, Clinical Informatics and Medical Statistics Research Center, Chang Gung University, 259 Wen-Hwa 1st Road, Kwei-Shan Tao-Yuan 333, Taiwan. jr@mail.cgu.edu.tw
} 
and the mMRC dyspnoea scale with regard to HRQoL. The St George's Respiratory Questionnaire (SGRQ), a diseasespecific questionnaire, and the World Health Organization Quality of Life-BREF (WHOQOL-BREF), a generic questionnaire, were used to measure HRQoL.

\section{METHODS}

A total of 328 patients were consecutively recruited from the pulmonology outpatient clinic of a teaching hospital in southern Taiwan between September 2007 and December 2009. The criteria for participation included an age of 40 years or more, and a diagnosis of COPD based on medical history and a post-bronchodilator $\mathrm{FEV}_{1}$ to forced vital capacity (FVC) ratio $\left(\mathrm{FEV}_{1} / \mathrm{FVC}\right)$ of $<0.7$. Patients with a history of asthma, active pulmonary tuberculosis, lung cancer or pulmonary resection were excluded. All of the recruited patients were clinically stable for at least four weeks prior to recruitment, without the need for a change in treatment regimens. The protocol was approved by the local ethics committee and all participating patients signed an informed consent form.

Patient demographic data such as age, gender, body mass index (BMI), level of education and smoking status were recorded. Any history of hypertension, diabetes, cardiovascular disease and sleep disorders, which are the most commonly reported comorbid conditions in both COPD patients ${ }^{(17-19)}$ and the general population, was also collected based on the International Classification of Diseases, Ninth Revision, codes in the local hospital information system. Hypertension, diabetes mellitus and sleep disorders include codes 250, 401-405 and 7805, respectively. Cardiovascular disease, denoted by a history of myocardial infarction, angina pectoris, ischaemic heart disease and heart failure, was coded as 410-414 and 428. Each patient was also asked to report on any symptoms experienced that were associated with chronic bronchitis (e.g. cough with sputum expectoration for at least three months per year over a period of two consecutive years). The number of exacerbations that occurred in the patients within the previous year was also acquired. The patients were categorised by GOLD spirometric classification (with cutoff points of $80 \%, 50 \%$ and $30 \% \mathrm{FEV}_{1}$ percentage predicted) and $\mathrm{mMRC}$ grades for statistical analysis.

Spirometry measurements were carried out according to the American Thoracic Society/European Respiratory Society guidelines, ${ }^{(20)}$ on the same day the quality of life questionnaires were collected, and using the HI701 spirometer (CHEST MI Inc, Tokyo, Japan). Post-bronchodilator tests were performed 15 minutes after the administration of $400 \mu$ g of salbutamol via a metred-dose inhaler with a spacer. Reference $\mathrm{FEV}_{1}$ and FVC values were calculated as previously described. ${ }^{(21)}$

The degree of dyspnoea was rated as previously described in the Mahler and Wells study ${ }^{(14)}$ - Grade 0: breathless with strenuous exercise; Grade 1: short of breath when hurrying on level ground or walking up a slight hill; Grade 2: walked slower than people of the same age on level ground, and experienced breathlessness or the need to stop to breathe when walking on level ground at their own pace; Grade 3: stop to breathe after walking about 100 yards, or after a few minutes on level ground; and Grade 4: too breathless to leave the house, or breathless when dressing or undressing. The degree of dyspnoea experienced by the patients was inquired immediately after the acquisition of patients' demographic data.

SGRQ is a disease-specific assessment tool designed to measure HRQoL in patients with asthma and COPD. ${ }^{(22)}$ The questionnaire consists of 50 items that are classified into three domains that measure symptoms, activity limitations and the psychosocial impacts related to the disease. Each domain was scored using a preset formula that individually weighs each option. A total score, which summarises the above three domains, was also produced. Scores can range from 0 to 100, with higher scores indicating poorer health status. We utilised a translated and validated version (Taiwan/Mandarin Chinese version) ${ }^{(23)}$ of SGRQ. WHOQOL is an international cross-culturally comparable quality of life assessment instrument developed by the World Health Organization. ${ }^{(24)}$ The WHOQOL-BREF is the abbreviated version of WHOQOL; it contains 26 items that belong to four domains (Physical, Psychological, Social and Environmental). Each item is derived from five options which represent scores ranging from 1 to 5; the three negatively-worded options were positively recoded during calculation. The mean score of each domain was transformed to a 0-100 scale, with higher scores representing an improved quality of life. The Taiwan version of WHOQOL-BREF, ${ }^{(25)}$ which was validated in Taiwan, was used in the present study.

Continuous variables were expressed as means and standard deviations, while categorical variables were expressed as numbers and percentages. The HRQoL scores of each domain were compared between the four stages of GOLD and the five grades of mMRC scale using analysis of variance (ANOVA). Scheffe post-hoc test was used for pairwise comparison, and multiple linear regression analysis was applied to explore the major determinants of HRQoL and exclude confounding factors. The impact of each parameter was expressed using an 'estimate' that represented the regression coefficient in continuous variables and the excess amount based on the controlled level in categorical variables. Statistical significance was established at $p<0.05$. Data were analysed using the Statistical Package for the Social Sciences version 15 (SPSS Inc, Chicago, Illinois, USA).

\section{RESULTS}

The baseline characteristics of the 328 enrolled patients are summarised in Table I. The mean patient age was 72.1 years (range 43-99 years) and most patients were male (89\%). Only $21 \%$ of the participants had a junior high school education or above. About a third (38.4\%) of the patients were smokers 
Table I. Baseline characteristics of patients $(n=328)$.

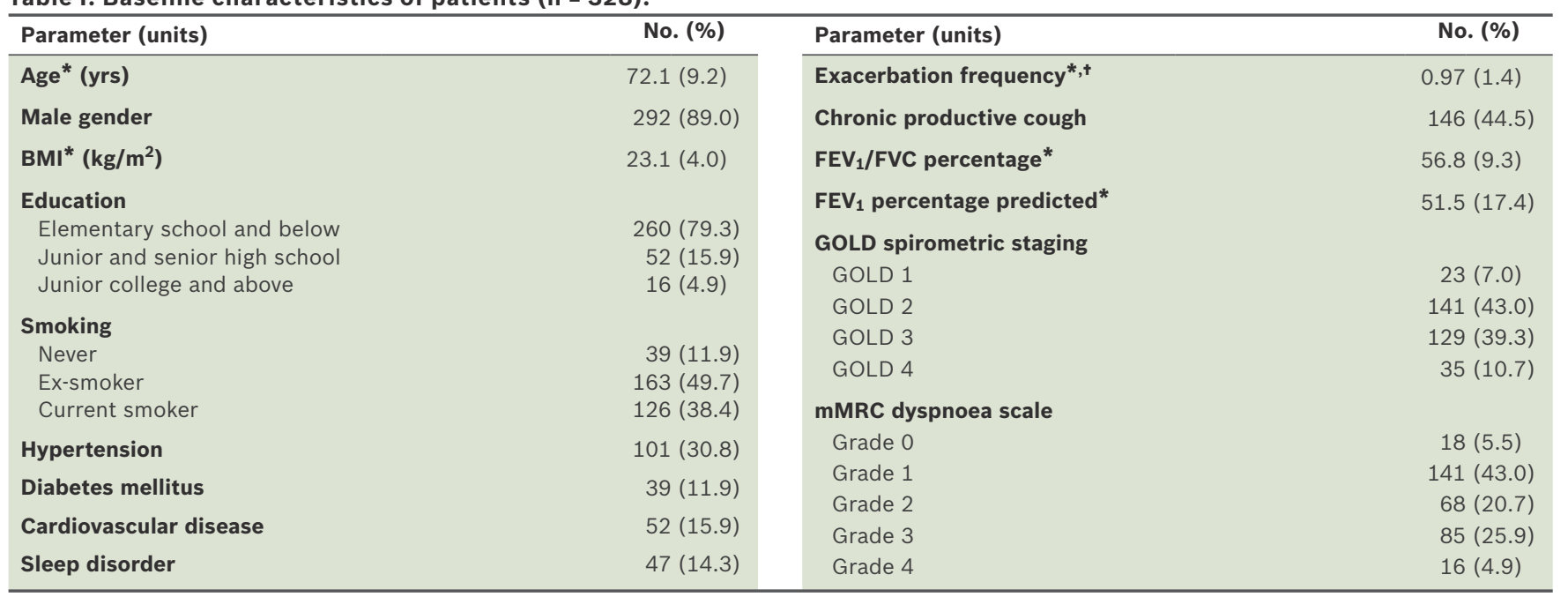

${ }^{*}$ Continuous variables are expressed as mean (standard deviation). ${ }^{\dagger}$ The number of exacerbations in the previous year.

BMI: body mass index; FEV 1 /FVC: forced expiratory volume in one second to forced vital capacity ratio; GOLD: Global Initiative for Chronic Obstructive Lung Disease; mMRC: modified Medical Research Council

Table II. Scores of each domain of SGRQ and WHOQOL-BREF, stratified according to the GOLD spirometric staging.

\begin{tabular}{|c|c|c|c|c|c|}
\hline \multirow[t]{2}{*}{ Index } & \multicolumn{4}{|c|}{ GOLD stages [Mean (SD)] } & \multirow[t]{2}{*}{ p-value } \\
\hline & Stage $1(n=23)$ & Stage $2(n=141)$ & Stage $3(n=129)$ & Stage $4(n=35)$ & \\
\hline \multicolumn{6}{|l|}{ SGRQ } \\
\hline Activity & $51.8(22.8)$ & $50.4(20.8)$ & $64.4(20.7)$ & $72.6(22.2)$ & $<0.001$ \\
\hline Impact & $43.7(17.4)$ & $41.3(17.3)$ & $48.4(15.8)$ & $54.1(15.6)$ & $<0.001$ \\
\hline Total & $46.7(16.8)$ & $44.8(16.5)$ & $54.0(15.0)$ & $61.0(16.0)$ & $<0.001$ \\
\hline \multicolumn{6}{|l|}{ WHOQOL-BREF } \\
\hline Physical & $47.5(18.4)$ & $46.5(18.5)$ & $43.9(18.9)$ & $33.2(17.7)$ & 0.002 \\
\hline Psychological & $54.3(20.5)$ & $58.8(19.3)$ & $58.1(19.2)$ & $50.1(19.2)$ & 0.097 \\
\hline Social & $64.1(15.2)$ & $64.3(17.7)$ & $64.2(16.3)$ & $60.0(18.0)$ & 0.588 \\
\hline Environmental & $73.3(14.4)$ & $71.6(15.7)$ & $71.2(14.1)$ & $68.6(12.7)$ & 0.643 \\
\hline
\end{tabular}

Significant differences between GOLD stages were found as follows: SGRQ Symptom: 1-4, 2-4; SGRQ Activity and Total: 1-4, 2-3, 2-4; SGRQ Impact: 2-3, 2-4; and in WHOQOL-BREF Physical: 1-4, 2-4, 3-4.

SD: standard deviation; SGRQ: St George's Respiratory Questionnaire; WHOQOL-BREF: World Health Organization Quality of Life (abbreviated version)

at the time the study was conducted, and about half $(44.5 \%)$ of the patients suffered from chronic productive cough. The patients experienced an average of about one exacerbation within the previous year. The prevalence of hypertension, diabetes mellitus, cardiovascular disease and sleep disorders was $30.8 \%, 11.9 \%, 15.9 \%$ and $14.3 \%$, respectively. The mean $\mathrm{FEV}_{1} / \mathrm{FVC}$ percentage was $56.8 \%$ and mean $\mathrm{FEV}_{1}$ percentage was $51.5 \%$. When stratified according to GOLD stages 1 to 4 , the proportion of patients was $7.0 \%, 43.0 \%, 39.3 \%$ and $10.7 \%$, respectively. When stratified according to the mMRC scale grades 0 to 4 , the proportion of patients was $5.5 \%, 43.0 \%$, $20.7 \%, 25.9 \%$ and $4.9 \%$, respectively.

The mean domain scores stratified according to GOLD stages are shown in Table II. Increasing GOLD stage was associated with lower HRQoL, reflected by the increasing SGRQ and decreasing WHOQOL-BREF domain scores. Significantly different scores between GOLD stages were found in all domains of SGRQ, but only in the Physical domain of WHOQOL-BREF. Post-hoc analysis showed that significant differences between GOLD stages were found mostly in SGRQ. There was significant overlap of the scores across GOLD stages, and the major changes between consecutive stages were found to occur between GOLD stages 2 and 3.

The mean domain scores stratified according to $\mathrm{mMRC}$ grades are presented in Table III. An increase in mMRC grade was accompanied by a deterioration in HRQoL, evidenced by increased SGRQ and reduced WHOQOL-BREF domain scores. All domain scores differed significantly among mMRC grades, implying that the mMRC scale had a better discriminative capacity than GOLD staging in HRQoL. Posthoc analysis showed significant differences in more domains of the two questionnaires among mMRC grades than among GOLD stages. There was much less overlap of the scores across mMRC grades, and most of the changes occurred evenly throughout all the grades.

Table IV summarises the results of the multiple linear regression analysis that evaluated each parameter's impact on SGRQ. The mMRC scale showed a strong discriminative capability in each of the domains of SGRQ $(p<0.001$, except for mMRC grade 1 in the Symptom domain $[p=0.008])$. Patients who experienced chronic productive cough and a higher number of exacerbations had poorer HRQoL, as reflected by the higher domain scores. 
Table III. Scores of each domain of SGRQ and WHOQOL-BREF, stratified according to the grades of the mMRC scale.

\begin{tabular}{|c|c|c|c|c|c|c|}
\hline \multirow[t]{2}{*}{ Index } & \multicolumn{5}{|c|}{ mMRC grades [Mean (SD)] } & \multirow[t]{2}{*}{ p-value } \\
\hline & Grade $0(n=18)$ & Grade 1 ( $n=141)$ & Grade $2(n=68)$ & Grade $3(n=85)$ & Grade $4(n=16)$ & \\
\hline \multicolumn{7}{|l|}{ SGRQ } \\
\hline Symptom & $32.4(17.6)$ & $44.9(17.9)$ & $52.0(19.2)$ & $57.4(17.0)$ & $59.3(17.0)$ & $<0.001$ \\
\hline Activity & $24.0(8.0)$ & $46.8(15.3)$ & $62.8(19.5)$ & $75.6(15.9)$ & $88.4(15.8)$ & $<0.001$ \\
\hline Impact & $23.7(10.6)$ & $38.7(14.4)$ & $49.2(15.0)$ & $55.3(14.0)$ & $65.0(12.2)$ & $<0.001$ \\
\hline Total & $25.3(8.3)$ & $42.2(12.8)$ & $53.9(14.3)$ & $62.0(12.2)$ & $71.3(11.4)$ & $<0.001$ \\
\hline \multicolumn{7}{|l|}{ WHOQOL-BREF } \\
\hline Physical & $63.7(12.4)$ & $50.6(15.8)$ & $40.6(17.8)$ & $35.1(18.7)$ & $27.2(15.8)$ & $<0.001$ \\
\hline Psychological & $78.4(15.0)$ & $59.7(17.5)$ & $54.4(21.0)$ & $52.9(18.7)$ & $47.1(18.8)$ & $<0.001$ \\
\hline Social & $75.0(12.9)$ & $66.6(17.2)$ & $60.5(17.4)$ & $61.0(16.0)$ & $55.5(13.7)$ & $<0.001$ \\
\hline Environmental & $78.7(14.3)$ & $72.8(14.3)$ & $70.5(14.8)$ & $69.0(14.2)$ & $64.3(16.4)$ & 0.017 \\
\hline
\end{tabular}

Significant differences among mMRC grades were found as follows: SGRQ Symptom: 0-2, 0-3, 0-4, 1-3; SGRQ Activity and Total: all except 3-4; SGRQ Impact: all except 2-3 and 3-4; WHOQOL-BREF Physical: all except 0-1, 2-3, 2-4, 3-4; WHOQOL-BREF Psychological: 0-1, 0-2, 0-3, 0-4; and WHOQOL-BREF Social: 0-2, 0-3, 0-4.

mMRC: modified Medical Research Council; SD: standard deviation; SGRQ: St George's Respiratory Questionnaire; WHOQOL-BREF: World Health Organization Quality of Life (abbreviated version)

Table IV. Multiple linear regression to assess each parameter's impact on HRQoL as measured by SGRQ.

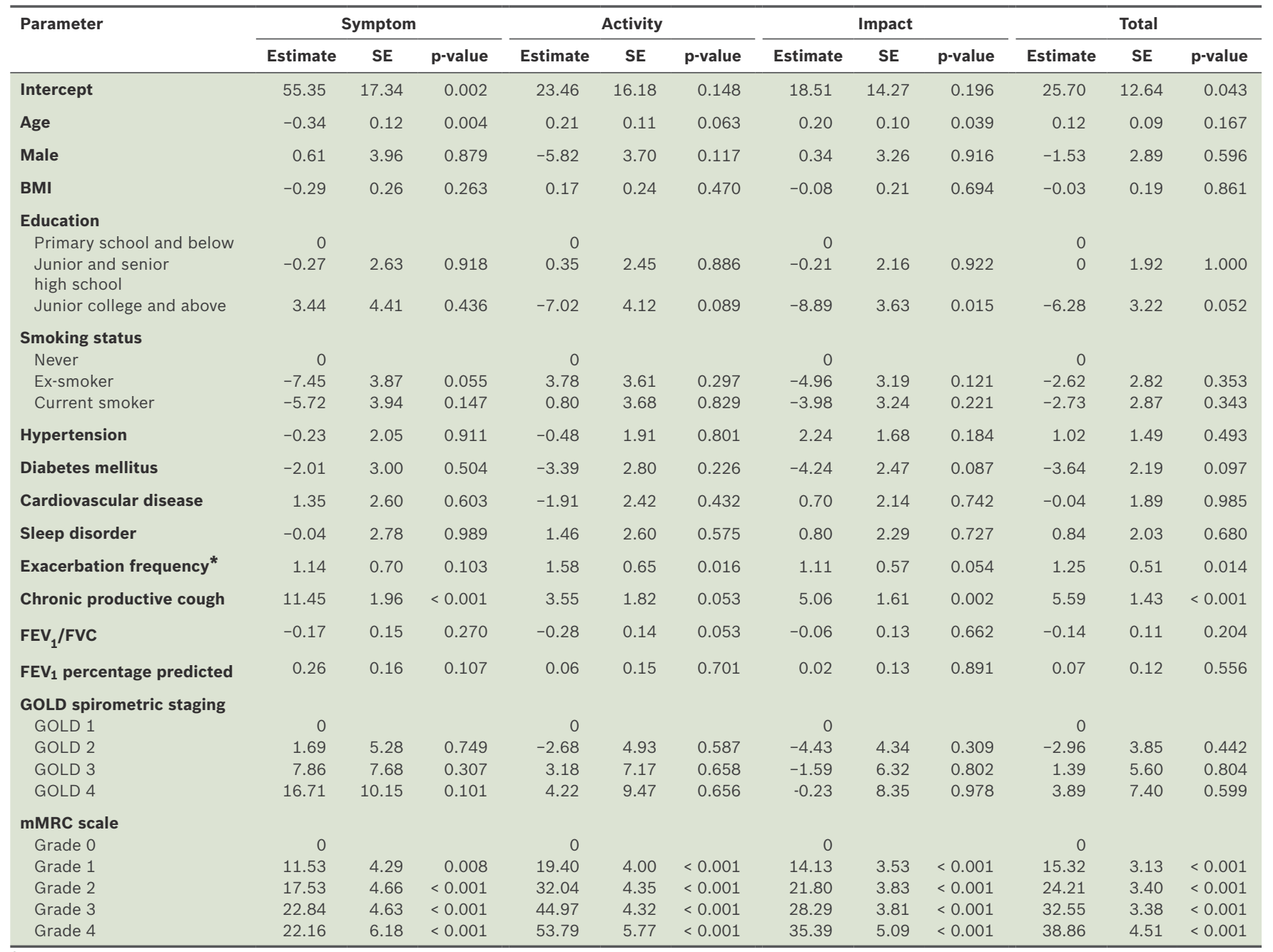

*The number of exacerbations in the previous year.

BMI: body mass index; FEV 1 /FVC: forced expiratory volume in 1 second to forced vital capacity ratio; GOLD: Global Initiative for Chronic Obstructive Lung Disease; HRQoL: health-related quality of life; mMRC: modified Medical Research Council; SE: standard error; SGRQ: St George's Respiratory Questionnaire

Age and education had sporadic or discordant effects on the Symptom and Impact domains, but not on the Activity and Total domains. These trends did not change when $F E V_{1} / F V C, F E V_{1}$ percentage or both were removed from the regression model.

The results of the multiple linear regression evaluating each parameter's impact on WHOQOL-BREF are reported in Table V. The discriminative capability of the mMRC scale remained significant in each of the four domains of WHOQOLBREF, except in mMRC grade 1 in the Social and Environmental domains. Aging had a negative effect on the Physical domain. Patients with sleep disorders had poorer HRQoL, as reflected by lower domain scores. These trends did not change when $\mathrm{FEV}_{1} / \mathrm{FVC}, \mathrm{FEV}_{1}$ percentage, or both were removed from the regression model. 
Table V. Multiple linear regression to assess each parameter's impact on HRQOL as measured by WHOQOL-BREF.

\begin{tabular}{|c|c|c|c|c|c|c|c|c|c|c|c|c|}
\hline \multirow[t]{2}{*}{ Parameter } & \multicolumn{3}{|c|}{ Physical } & \multicolumn{3}{|c|}{ Psychological } & \multicolumn{3}{|c|}{ Social } & \multicolumn{3}{|c|}{ Environmental } \\
\hline & Estimate & SE & p-value & Estimate & SE & p-value & Estimate & SE & p-value & Estimate & SE & p-value \\
\hline Intercept & 93.97 & 16.29 & $<0.001$ & 73.11 & 19.07 & $<0.001$ & 90.34 & 17.50 & $<0.001$ & 70.81 & 15.18 & $<0.001$ \\
\hline Age & -0.51 & 0.11 & $<0.001$ & -0.03 & 0.13 & 0.810 & -0.21 & 0.12 & 0.078 & 0.13 & 0.10 & 0.212 \\
\hline Male & -0.90 & 3.72 & 0.808 & 1.44 & 4.36 & 0.742 & -5.52 & 4.00 & 0.169 & 1.35 & 3.47 & 0.698 \\
\hline BMI & -0.41 & 0.24 & 0.089 & -0.36 & 0.28 & 0.201 & -0.14 & 0.26 & 0.603 & -0.09 & 0.22 & 0.684 \\
\hline Primary school and below & 0 & & & 0 & & & 0 & & & 0 & & \\
\hline $\begin{array}{l}\text { Junior and senior } \\
\text { high school }\end{array}$ & 5.18 & 2.47 & 0.037 & 5.56 & 2.89 & 0.055 & 2.46 & 2.65 & 0.356 & 3.29 & 2.30 & 0.154 \\
\hline Junior college and above & 4.60 & 4.15 & 0.268 & 1.74 & 4.86 & 0.721 & 2.11 & 4.46 & 0.637 & -0.80 & 3.86 & 0.836 \\
\hline \multicolumn{13}{|l|}{ Smoking status } \\
\hline Never & 0 & & & 0 & & & 0 & & & 0 & & \\
\hline Ex-smoker & 6.02 & 3.64 & 0.099 & 5.83 & 4.26 & 0.172 & 5.42 & 3.91 & 0.166 & 3.46 & 3.39 & 0.308 \\
\hline Current smoker & 8.81 & 3.70 & 0.018 & 7.75 & 4.33 & 0.075 & 5.23 & 3.98 & 0.190 & 3.83 & 3.45 & 0.267 \\
\hline Diabetes mellitus & 2.91 & 2.82 & 0.302 & 1.31 & 3.30 & 0.692 & 0.73 & 3.03 & 0.811 & 2.73 & 2.63 & 0.300 \\
\hline Cardiovascular disease & -2.38 & 2.44 & 0.329 & -0.54 & 2.86 & 0.852 & 0.33 & 2.62 & 0.901 & 1.75 & 2.27 & 0.443 \\
\hline Sleep disorder & -8.11 & 2.61 & 0.002 & -8.93 & 3.06 & 0.004 & -0.84 & 2.81 & 0.764 & -5.58 & 2.44 & 0.023 \\
\hline Exacerbation frequency* & -0.58 & 0.65 & 0.374 & -0.36 & 0.76 & 0.642 & -0.66 & 0.70 & 0.347 & -0.25 & 0.61 & 0.676 \\
\hline Chronic productive cough & -2.34 & 1.84 & 0.203 & -1.82 & 2.15 & 0.399 & 0.14 & 1.97 & 0.945 & 1.63 & 1.71 & 0.342 \\
\hline $\mathrm{FEV}_{1} / \mathrm{FVC}$ & 0.16 & 0.14 & 0.275 & 0.17 & 0.17 & 0.316 & 0.01 & 0.16 & 0.946 & 0.09 & 0.13 & 0.527 \\
\hline FEV $_{1}$ percentage predicted & -0.03 & 0.15 & 0.856 & -0.09 & 0.17 & 0.602 & 0.02 & 0.16 & 0.920 & -0.09 & 0.14 & 0.500 \\
\hline \multicolumn{13}{|l|}{ GOLD spirometric staging } \\
\hline GOLD 1 & 0 & & & 0 & & & 0 & & & 0 & & \\
\hline GOLD 2 & 0.58 & 4.96 & 0.906 & 4.60 & 5.81 & 0.429 & 0.81 & 5.33 & 0.880 & -3.60 & 4.62 & 0.437 \\
\hline GOLD 3 & 1.11 & 7.21 & 0.878 & 5.57 & 8.45 & 0.510 & 2.57 & 7.75 & 0.740 & -3.93 & 6.72 & 0.559 \\
\hline GOLD 4 & -7.87 & 9.54 & 0.410 & -1.98 & 11.17 & 0.860 & 0.80 & 10.25 & 0.938 & -6.40 & 8.89 & 0.472 \\
\hline \multicolumn{13}{|l|}{ mMRC scale } \\
\hline Grade 2 & -17.10 & 4.38 & $<0.001$ & -22.98 & 5.12 & $<0.001$ & -13.67 & 4.70 & 0.004 & -9.13 & 4.08 & 0.026 \\
\hline Grade 3 & -21.70 & 4.35 & $<0.001$ & -23.64 & 5.10 & $<0.001$ & -13.31 & 4.68 & 0.005 & -10.31 & 4.06 & 0.011 \\
\hline Grade 4 & -25.15 & 5.81 & $<0.001$ & -27.64 & 6.80 & $<0.001$ & -17.16 & 6.24 & 0.006 & -14.61 & 5.41 & 0.007 \\
\hline
\end{tabular}

*The number of exacerbations in the previous year.

BMI: body mass index; FEV 1 /FVC: forced expiratory volume in 1 second to forced vital capacity ratio; GOLD: Global Initiative for Chronic Obstructive Lung Disease; HRQoL: health-related quality of life; mMRC: modified Medical Research Council; SE: standard error; WHOQOL-BREF: World Health Organization Quality of Life (abbreviated version)

\section{DISCUSSION}

To the best of our knowledge, the present study is the first study to investigate the determinant factors of HRQoL in patients with COPD of all levels of severity, using both disease-specific and generic questionnaires. We found inconsistencies between the GOLD spirometric classification of COPD and HRQoL, as measured by both SGRQ and WHOQOL-BREF. We also found that the mMRC scale had better discriminative capacity than the GOLD classification in the evaluation of HRQoL, as evidenced by the results of both the ANOVA and multiple linear regression models.

Although COPD is characterised by airflow limitations, it involves several systemic components. ${ }^{(1,26,27)} \mathrm{HRQoL}$, a comprehensive and widely accepted measure of the impact of disease on a patient's quality of life, may help medical staff optimise treatment and help patients plan for the increasing disability associated with the disease. ${ }^{(28)}$ Questionnaires such as SGRQ and WHOQOL-BREF are good tools for gauging HRQoL, but their use in clinical practice is limited due to time constraints. Spirometry provides a useful measure of the pathological changes in COPD, but poorly correlates with some important outcomes. ${ }^{(27)}$ As shown in the present study, as well as in previously published data, there is considerable heterogeneity in HRQoL impairment within each GOLD stage of severity. ${ }^{(3)}$ It has also been demonstrated that the level of dyspnoea may be a more accurate measure of the HRQoL of patients with COPD compared to measurement by categorisation of spirometry results. ${ }^{(12)}$

The MRC dyspnoea scale has been utilised for many years to grade disability resulting from breathlessness. ${ }^{(14,29)}$ It is short, simple to execute and can be easily performed in clinical settings. Its five consecutive questions describe patients' daily activities, and this explains why it correlates best with the SGRQ Activity and WHOQOL-BREF Physical domains, as was observed in the present study. The mMRC scale also demonstrated excellent discriminative capabilities in other domains such as the Impact, Psychological and Social domains, thus proving to be a good indicator of HRQoL.

When categorised using GOLD stages, the participants' HRQoL showed considerable variation and was not well distinguished. Nevertheless, the domain scores of SGRQ were significantly increased between stages 2 and 3; this was not 
observed between other consecutive stages. Our findings are consistent with reports by Jones et $\mathrm{al}^{(3)}$, Antonelli-Incalzi et $\mathrm{al}^{(11)}$ and Hajiro et $\mathrm{al}^{(12)}$, which found that there was dramatic deterioration of HRQoL when patients' $\mathrm{FEV}_{1}$ dropped below $50 \%$ of the predicted normal values. The $50 \%$ predicted $\mathrm{FEV}_{1}$ represents a critical point of $\mathrm{HRQoL}$ and is a likely an indicator of general health status. Therefore, therapies for COPD, either pharmacological or non-pharmacological, should be started and enforced early (before $50 \% \mathrm{FEV}_{1}$ is reached), considering the potential for irreversible impairment, which is ultimately less treatable.

It has been demonstrated that the deterioration of lung function is more prominent in the early stages of COPD (i.e. in GOLD stages 1 and 2). ${ }^{(30)}$ Symptomatic patients with early COPD demonstrate rapid disease progression compared to asymptomatic patients. ${ }^{(31)}$ In the current study, $87.0 \%, 91.5 \%$, $97.7 \%$ and $100.0 \%$ of our participants who were classified as GOLD stage 1 through 4 had mMRC grades 1, 2, 3 and 4, respectively. In other words, most patients were symptomatic and somewhat restricted in their daily activities. Compared to GOLD staging, we were able to detect the deterioration of HRQoL earlier using mMRC grading. Early detection enabled us to take appropriate and effective actions to relieve patients' symptoms, improve patients' exercise tolerance, prevent exacerbations and slow down disease progression.

The present study's multiple linear regression analysis showed that other parameters were also determining factors of HRQoL. 'Chronic productive cough', a symptom that is routinely reported in both in- and outpatient settings, had a negative effect on the HRQoL of patients with COPD. This is noteworthy because although the symptoms 'cough' and 'sputum production' were mentioned in only five of the 50 items of SGRQ, these symptoms showed extensive influence on most of the SGRQ domains. Exacerbations of COPD also showed an adverse influence on HRQoL, compatible with the findings of previous studies. ${ }^{(32,33)}$ Sleep disorders, especially sleep apnoea, are associated with increased mortality and hospitalisation of COPD patients, ${ }^{(19)}$ and in our study, sleep disorders also showed a detrimental impact on HRQoL. Aging also had a negative effect on HRQoL in the SGRQ Impact and WHOQOL-BREF Physical domains, but showed otherwise in the SGRQ Symptom domain. This discrepancy may be attributed to a blunted perception of dyspnoea in older patients with COPD. ${ }^{(34)}$ Smoking status and level of education revealed sporadic and borderline effects, and may be of little significance since each of the item involves only one domain of the questionnaires. Further studies are needed to address the roles of age, smoking and level of education with regard to HRQoL.

The aim of the present study was not to abolish the importance of pulmonary function testing in the diagnosis and treatment of COPD. Instead, the study was designed to discover determinant factors of HRQoL other than lung function and GOLD staging. Spirometry is still the cornerstone for the diagnosis of COPD, but it is not the only tool in the preliminary evaluation of disease severity. As mentioned by Hajiro et al, categorising patients according to their level of dyspnoea (e.g. using the mMRC scale) may help explain changes in HRQoL, which is often used as an endpoint in clinical trials or as a marker of treatment effects. ${ }^{(12)}$

There are some limitations in the present study. First, the number of participants in GOLD stages 1 and 4 were significantly lesser than those in GOLD stages 2 and 3. This unbalanced representation of patients has also been observed in the

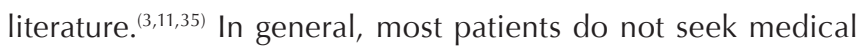
treatment until they are symptomatic, and thus present with relatively bad lung function at the time of diagnosis. On the other hand, patients diagnosed with severely impaired lung function (i.e. GOLD stage 4) are frequently hospitalised due to acute exacerbation, and are thus prone to become dependent on mechanical ventilation. These patients are also more inclined to have cardiovascular events and lung cancer, which increases mortality. We postulate that these are the reasons why we had fewer patients in GOLD stages 1 and 4 . Furthermore, our participants were recruited from an outpatient clinic. A thorough and well-designed survey on community populations may be able to overcome this limitation. The second limitation of our study was that most of our participants were male. This may be due to the fact that in Taiwan, a large proportion $(55 \%-60 \%)$ of men are smokers while only a small proportion $(3 \%-4 \%)$ of women smoke, ${ }^{(36)}$ and cigarette smoking is a well-known risk factor for COPD. Although multiple linear regression analysis did not show significant difference in HRQoL between men and women with COPD, we believe that further studies with a more balanced sample of men and women are needed.

In conclusion, the mMRC dyspnoea scale is a concise and practical tool to assess the HRQoL of patients with COPD in daily clinical practice. Compared to GOLD staging, it demonstrates better discriminative validity in evaluating HRQoL and is able to detect impaired health status earlier. In addition to decreased lung function, chronic productive cough, sleep disorders and frequent exacerbations also have negative effects on HRQoL. Therefore, these factors should be taken into consideration when HRQoL is included as a measurement of patients' baseline condition or treatment effects.

\section{ACKNOWLEDGEMENTS}

We would like to thank Prof Paul W Jones (Saint George's Hospital Medical School, London, UK) for providing us with the Taiwan/Mandarin Chinese version of the St George's Respiratory Questionnaire, and Prof Grace Yao (National Taiwan University, Taipei, Taiwan) for granting us permission to use the Taiwanese version of the WHOQOL-BREF. We also appreciate the helpful comments from Prof Jung-Der Wang (National Taiwan University, Taipei, Taiwan). 


\section{REFERENCES}

1. Global Initiative for Chronic Obstructive Lung Disease. Global strategy for the diagnosis, management, and prevention of chronic obstructive pulmonary disease. (Updated 2010). Available at: http://www.goldcopd. org/uploads/users/files/GOLDReport_April112011.pdf. Accessed: August 31, 2011.

2. Marin JM, Cote CG, Diaz O, et al. Prognostic assessment in COPD: Health related quality of life and the BODE index. Respir Med 2011; 105:916-21.

3. Jones PW, Brusselle G, Dal Negro RW, et al. Health-related quality of life in patients by COPD severity within primary care in Europe. Respir Med 2011; 105:57-66.

4. Calverley PM, Anderson JA, Celli B, et al. Salmeterol and fluticasone propionate and survival in chronic obstructive pulmonary disease. $\mathrm{N}$ Engl J Med 2007; 356:775-89.

5. Tashkin DP, Celli B, Senn S, et al. A 4-year trial of tiotropium in chronic obstructive pulmonary disease. N Engl J Med 2008; 359:1543-54.

6. Fishman A, Martinez F, Naunheim K, et al. A randomized trial comparing lung-volume-reduction surgery with medical therapy for severe emphysema. N Engl J Med 2003; 348:2059-73.

7. Ries AL, Kaplan RM, Limberg TM, Prewitt LM. Effects of pulmonary rehabilitation on physiologic and psychosocial outcomes in patients with chronic obstructive pulmonary disease. Ann Intern Med 1995; 122:823-32.

8. Domingo-Salvany A, Lamarca R, Ferrer M, et al. Health-related quality of life and mortality in male patients with chronic obstructive pulmonary disease. Am J Respir Crit Care Med 2002; 166:680-5.

9. Fan VS, Curtis JR, Tu SP, McDonell MB, Fihn SD. Using quality of life to predict hospitalization and mortality in patients with obstructive lung diseases. Chest 2002; 122:429-36.

10. Engstrom CP, Persson LO, Larsson S, Sullivan M. Health-related quality of life in COPD: why both disease-specific and generic measures should be used. Eur Respir J 2001; 18:69-76.

11. Antonelli-Incalzi R, Imperiale C, Bellia V, et al. Do GOLD stages of COPD severity really correspond to differences in health status? Eur Respir J 2003; 22:444-9.

12. Hajiro T, Nishimura K, Tsukino M, Ikeda A, Oga T, Izumi T. A comparison of the level of dyspnea vs disease severity in indicating the health-related quality of life of patients with COPD. Chest 1999; 116:1632-7.

13. Medinas Amoros M, Mas-Tous C, Renom-Sotorra F, et al. Health-related quality of life is associated with COPD severity: a comparison between the GOLD staging and the BODE index. Chron Respir Dis 2009; 6:75-80.

14. Mahler DA, Wells CK. Evaluation of clinical methods for rating dyspnea. Chest 1988; 93:580-6.

15. Bestall JC, Paul EA, Garrod R, et al. Usefulness of the Medical Research Council (MRC) dyspnoea scale as a measure of disability in patients with chronic obstructive pulmonary disease. Thorax 1999; 54:581-6.

16. Oga T, Nishimura K, Tsukino M, et al. Longitudinal deteriorations in patient reported outcomes in patients with COPD. Respir Med 2007; 101:146-53.

17. Mannino DM, Thorn D, Swensen A, Holguin F. Prevalence and outcomes of diabetes, hypertension and cardiovascular disease in COPD. Eur Respir J 2008; 32:962-9.

18. Nizet TA, van den Elshout FJ, Heijdra YF, et al. Survival of chronic hypercapnic COPD patients is predicted by smoking habits, comorbidity, and hypoxemia. Chest 2005; 127:1904-10.

19. Marin JM, Soriano JB, Carrizo SJ, Boldova A, Celli BR. Outcomes in patients with chronic obstructive pulmonary disease and obstructive sleep apnea: the overlap syndrome. Am J Respir Crit Care Med 2010; 182:325-31.

20. Miller MR, Hankinson J, Brusasco V, et al. Standardisation of spirometry. Eur Respir J 2005; 26:319-38.

21. Berglund E, Birath G, Bjure J, et al. Spirometric studies in normal subjects. I. Forced expirograms in subjects between 7 and 70 years of age. Acta Med Scand 1963; 173:185-92

22. Jones PW, Quirk FH, Baveystock CM, Littlejohns P. A self-complete measure of health status for chronic airflow limitation. The St. George's Respiratory Questionnaire. Am Rev Respir Dis 1992; 145:1321-7.

23. Wang KY, Chiang $\mathrm{CH}$, Maa SH, Shau WY, Tarn YH. Psychometric assessment of the Chinese language version of the St. George's Respiratory Questionnaire in Taiwanese patients with bronchial asthma. J Formos Med Assoc 2001; 100:455-60.

24. The WHOQOL Group. The World Health Organization Quality of Life Assessment (WHOQOL): development and general psychometric properties. Soc Sci Med. 1998; 46:1569-85.

25. Yao G, Chung CW, Yu CF, Wang JD. Development and verification of validity and reliability of the WHOQOL-BREF Taiwan version. J Formos Med Assoc 2002;101:342-51.

26. Agusti A. Thomas a. Neff lecture. Chronic obstructive pulmonary disease: a systemic disease. Proc Am Thorac Soc 2006; 3:478-81.

27. Papaioannou AI, Loukides S, Gourgoulianis KI, Kostikas K. Global assessment of the COPD patient: time to look beyond FEV1? Respir Med 2009: 103:650-60.

28. Jenkins C, Rodriguez-Roisin R. Quality of life, stage severity and COPD. Eur Respir J 2009; 33:953-5.

29. Fletcher CM, Elmes PC, Fairbairn AS, Wood CH. The significance of respiratory symptoms and the diagnosis of chronic bronchitis in a working population. Br Med J 1959; 2:257-66.

30. Jones R, Ostrem A. Optimising pharmacological maintenance treatment for COPD in primary care. Prim Care Respir J 2011; 20:33-45.

31. Bridevaux PO, Gerbase MW, Probst-Hensch NM, et al. Long-term decline in lung function, utilisation of care and quality of life in modified GOLD stage 1 COPD. Thorax 2008; 63:768-74.

32. Seemungal TA, Donaldson GC, Paul EA, et al. Effect of exacerbation on quality of life in patients with chronic obstructive pulmonary disease. Am J Respir Crit Care Med 1998; 157:1418-22.

33. Spencer S, Calverley PM, Burge PS, Jones PW. Impact of preventing exacerbations on deterioration of health status in COPD. Eur Respir 2004; 23:698-702.

34. Rutgers SR, ten Hacken NH, Koeter GH, Postma DS. Borg scores before and after challenge with adenosine $5^{\prime}$-monophosphate and methacholine in subjects with COPD and asthma. Eur Respir J 2000; 16:486-90.

35. Jones RC, Donaldson GC, Chavannes NH, et al. Derivation and validation of a composite index of severity in chronic obstructive pulmonary disease: the DOSE Index. Am J Respir Crit Care Med 2009; 180:1189-95.

36. Annual report of tobacco, alcohol consumption investigation in Taiwan area. Taipei, Taiwan: Bureau of Tobacco and Alcohol Monopoly, Taiwan Provincial Government, 1993. 